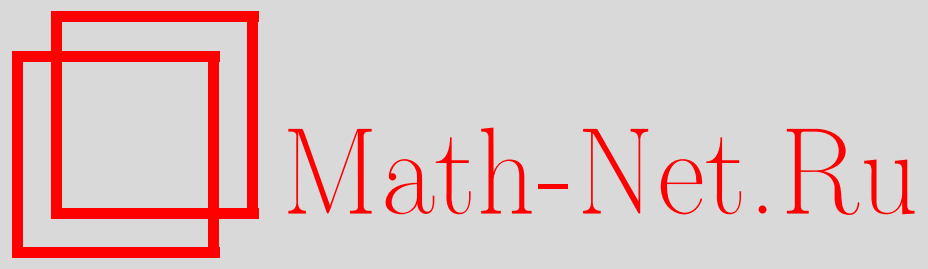

Б. О. Василевский, Достаточное условие несингулярности дискретного конечнозонного при одной энергии двумерного оператора Шрёдингера на квад-графе, Функи. анализ и его прил., 2015, том 49, выпуск 3, 65-70

DOI: https://doi.org/10.4213/faa3199

Использование Общероссийского математического портала MathNet.Ru подразумевает, что вы прочитали и согласны с пользовательским соглашением

http://www.mathnet.ru/rus/agreement

Параметры загрузки:

IP : 3.80 .181 .102

26 апреля 2023 г., 18:36:00

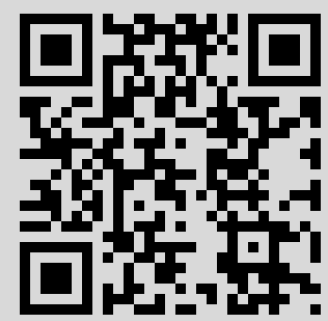


Можно также вычислить матрицы $Q^{-}$и $Q^{+}$:

$$
\begin{aligned}
& Q^{-}=\left(\begin{array}{cccccc}
0 & 0 & 0 & 0 & 0 & 0 \\
0 & \frac{1}{2}(\cos t)^{2} & \frac{1}{2} \sin t \cos t & 0 & 0 & 0 \\
0 & \frac{1}{2} \cos t \sin t & \frac{1}{2}(\sin t)^{2} & 0 & 0 & 0 \\
0 & 0 & 0 & \frac{1}{2}(\sin t)^{2} & -\frac{1}{2} \cos t \sin t & 0 \\
0 & 0 & 0 & -\frac{1}{2} \cos t \sin t & \frac{1}{2}(\cos t)^{2} & 0 \\
0 & 0 & 0 & 0 & 0 & 0
\end{array}\right), \\
& Q^{+}=\left(\begin{array}{cccccc}
1 & 0 & 1 & 0 & 1 & 0 \\
0 & 1 & 0 & 1 & 0 & 1 \\
1 & 0 & 1 & 0 & 1 & 0 \\
0 & 1 & 0 & 1 & 0 & 1 \\
1 & 0 & 1 & 0 & 1 & 0 \\
0 & 1 & 0 & 1 & 0 & 1
\end{array}\right)
\end{aligned}
$$

Теперь можно вычислить матрицы $K_{n}$. Ненулевые собственные числа матрицы $\rho_{n}$ равны собственным числам матрицы $K_{n}$ и равны $\frac{1}{4}+\frac{1}{4}(-\cos 2 t)^{n+1}, \quad \frac{1}{4}+\frac{1}{4}(-\cos 2 t)^{n+1}, \quad \frac{1}{4}-\frac{1}{4}(-\cos 2 t)^{n+1}, \quad \frac{1}{4}-\frac{1}{4}(-\cos 2 t)^{n+1}$.

Предел энтропий фон Неймана равен $\ln 4$.

\title{
ЛитерАТУРА
}

[1] Л. Аккарди, Функц. анализ и его прил., 9:1 (1975), 1-8. [2] L. Accardi, F. Fidaleo, Ann. Mat. Pura Appl., 184:3 (2005), 327-346. [3] M. Fannes, B. Nachtergaele, R. F. Werner, Comm. Math. Phys., 144:3 (1992), 443-490. [4] З. И. Бежаева, В. И. Оселедец, Зап. научн. сем. ПОМИ, 326 (2005), 28-47. [5] A. Jamiołkowski, Rep. Mathematical Phys., 3:4 (1972), 275-278. [6] M. D. Choi, Linear Algebra Appl., 10 (1975), 285-290.

Московский институт электроники и математики Национальный исследовательский университет

«Высшая школа экономики»

e-mail: zbejaeva@hse.ru

Финансовый университет при Правительстве РФ Московский государственный университет

им. М. В. Ломоносова

e-mail: oseled@gmail.com
Поступила в редакцию 30 ноября 2014 г.

УДК 514.84

\section{Достаточное условие несингулярности дискретного конечнозонного при одной энергии двумерного оператора Шрёдингера на квад-графе*}

\author{
(c) 2015. Б. О. ВАСИлЕВСКИй
}

Интегрируемость двумерного стационарного конечнозонного оператора Шрёдингера при фиксированной энергии была установлена в работе Дубровина, Кричевера и Новикова [1]. Большой интерес вызывает задача построения ин-

* Работа выполнена при поддержке гранта Правительства Российской федерации 2010220-01-077.

3 Функциональный анализ и его приложения, т. 49, вып. 3 
тегрируемых дискретизаций этого оператора. Интегрируемая гиперболическая дискретизация конечнозонного оператора на квадратной решетке была найдена Кричевером [2]. Эллиптическая дискретизация как специальная редукция гиперболической была построена в статье Доливы, Гриневича, Нешпорского и Сантини [3]. Эта редукция оказалась аналогичной найденной Веселовым и Новиковым [4] для непрерывного случая.

Построенные в [3] решения отвечали голоморфной инволюции на кривой с двумя неподвижными точками. Из работы Кричевера и Грушевского [5], в частности, следует, что общие решения отвечают инволюции без неподвижных точек, а решения из [3] являются специальными. Тем не менее именно этот специальный случай мы будем обсуждать. Результат данной работы для инволюций с произвольным количеством неподвижных точек - тема для дальнейших исследований.

Напомним основные определения из теории дискретного комплексного анализа. Подробности можно найти в [7]. Квад-графом называется клеточное разбиение плоскости $\mathbb{C}$, каждая грань которого является четырехугольником. Это интересное обобщение квадратной решетки на плоскости. Существует непустой класс подграфов, допускающих такое вложение к комплексную плоскость, при котором каждая грань является ромбом [7, теорема 6]. Если вложение является квазикристаллическим, то соответствующий квад-граф можно представить в виде подкомплекса стандартного клеточного комплекса на $\mathbb{Z}^{d}$ с клетками размерности не более двух [7, гл. 3]. Теорема 6 из [7] дает также критерий представимости двумерного подкомплекса решетки $\mathbb{Z}^{d}$ в виде ромбовидного квазикристаллического вложения квад-графа в $\mathbb{C}$. Далее везде мы требуем, чтобы данный квад-граф $\mathscr{D}$ представлялся в виде двумерного подкомплекса решетки $\mathbb{Z}^{d}$. Будем использовать следующие обозначения: $V(\mathscr{D})-$ множество вершин графа $\mathscr{D}, E(\mathscr{D})$ - множество его ребер, $F(\mathscr{D})$ - множество его положительно ориентированных граней, причем ориентация индуцируется с плоскости $\mathbb{C}$. Отображение вершин графа в узлы решетки при таком представлении обозначим через $\mathbf{n}: V(\mathscr{D}) \rightarrow \mathbb{Z}^{d}$.

Пусть на диагоналях граней данного квад-графа $\mathscr{D}$ определена комплекснозначная функция $\nu$, причем для двух диагоналей $e, e^{*}$ одной грани значения функции обратны: $\nu(e) \nu\left(e^{*}\right)=1$. Мы будем назвать такую функцию $\nu$ весовой функцией. Комплекснозначная функция $f: V(\mathscr{D}) \rightarrow \mathbb{C}$ на вершинах квад-графа называется дискретной голоморфной (относительно $\nu$ ), если для любой положительно ориентированной грани $\left(x_{0}, y_{0}, x_{1}, y_{1}\right)$

$$
\frac{f\left(y_{1}\right)-f\left(y_{0}\right)}{f\left(x_{1}\right)-f\left(x_{0}\right)}=i \nu\left(x_{0}, x_{1}\right)=-\frac{1}{i \nu\left(y_{0}, y_{1}\right)} .
$$

Квад-граф $\mathscr{D}$ является двудольным, в каждой грани одной доле принадлежат противоположные вершины [7, гл. 2]. Таким образом строятся два графа $\mathscr{G}$, $\mathscr{G}^{*}$, являющиеся клеточными разбиениями плоскости $\mathbb{C}$, грани которых имеют произвольное число вершин. Ребра каждого из графов являются диагоналями граней квад-графа $\mathscr{D}$. Определим оператор Лапласа на функциях $f: V(\mathscr{G}) \rightarrow \mathbb{C}$ по формуле

$$
(\Delta f)\left(x_{0}\right)=\sum_{x \sim x_{0}} \nu\left(x_{0}, x\right)\left(f(x)-f\left(x_{0}\right)\right),
$$


где суммирование проходит по всем соседним с $x_{0}$ вершинам в $\mathscr{G}$. Функция $f$ называется дискретной гармонической (относительно $\nu$ ), если $\Delta f \equiv 0$.

Обсудим определение несингулярности оператора $\Delta$. Выберем в графе $\mathscr{G}$ простой цикл $p=\left(x_{1}, \ldots, x_{n}, x_{1}\right)$. Он делит $V(\mathscr{G}) \backslash p$ на две части, выберем одну из них в качестве внутренней. Рассмотрим дискретную краевую задачу: продолжить функцию $f$ с вершин цикла $p$ на внутренние вершины так, чтобы внутри выполнялось равенство $\Delta f=0$. Если весовая функция принимает только положительные (либо только отрицательные) значения, то несложно показать выполнение дискретного аналога принципа максимума модуля, из которого следует единственность для краевой задачи. В связи с этим логично называть оператор $\Delta$ несингулярным, если соответствующая ему весовая функция $\nu: E(\mathscr{G}) \rightarrow \mathbb{R}$ принимает только значения одного знака.

Итак, пусть дан квад-граф $\mathscr{D}$, реализуемый в виде подкомплекса стандартного клеточного комплекса на $\mathbb{Z}^{d}$ с клетками размерности не более двух. Используя конечнозонный подход, аналогичный используемому в [8], мы строим по спектральным данным весовую функцию $\nu$. Далее мы представляем результат статьи - достаточное условие несингулярности соответствующего оператора Лапласа в терминах спектральных данных и отображения $\mathbf{n}$.

1. Многоточечная волновая функция. Мы приступаем к описанию того, как, используя конечнозонный подход, по отображению $\mathbf{n}$ построить весовую функцию $\nu$, через которую выписываются уравнения Коши-Римана на квадграфе $\mathscr{D}$, а также волновую функцию для этих уравнений. Весовая функция $\nu$, в свою очередь, определяется через обобщенные спектральные данные, о которых сейчас пойдет речь.

Описанная ниже конструкция является частным случаем построений, описанных еще в [8]. Кроме этого, она обобщает построения, сделанные в [2] и в [3]. А именно, в указанных работах рассматривается случай квадратной решетки на плоскости и $d=2$.

Рассмотрим компактную регулярную риманову поверхность $Г$ рода $g$. Фиксируем на ней точку нормировки $R_{1}, \gamma$-дивизор общего положения $\gamma_{1}, \ldots, \gamma_{g}$ и коллекцию из $d$ пар выделенных точек $A_{1}^{+}, A_{1}^{-}, \ldots, A_{d}^{+}, A_{d}^{-}$. Все точки должны быть попарно различны.

По теореме Римана-Роха для любого целочисленного вектора $\mathbf{m}=\left(n_{1}, \ldots, n_{d}\right)$ $\in \mathbb{Z}^{d}$ существует единственная функция $\Psi(\mathbf{m} ; \lambda), \lambda \in \Gamma$, такая, что: (1) при каждом $\mathbf{m}$ функция $\Psi$ является мероморфной функцией от $\lambda ;(2) \Psi$ имеет полюсы не более первого порядка в точках $\gamma_{1}, \ldots, \gamma_{g} ;(3)$ для каждого $j=1, \ldots, d$ функция $\Psi$ имеет полюс порядка не более чем $m_{j}$ в точке $A_{j}^{+}$и нуль по крайней мере порядка $m_{j}$ в точке $A_{j}^{-} ;(4)$ выполняется условие нормировки $\Psi\left(\mathbf{m} ; R_{1}\right) \equiv 1$. Будем называть $\Psi$ волновой функиией. Она естественно переносится на вершины $p \in V(\mathscr{D})$ графа: $\Psi(p, \lambda)=\Psi(\mathbf{n}(p) ; \lambda)$.

Напомним, что при отображении $\mathbf{n}$ каждое ребро графа $\mathscr{D}$ переходит в единичный отрезок, параллельный одной из осей координат в $\mathbb{Z}^{d}$. Ориентируем ребра графа $\mathscr{D}$ по возрастанию координаты. Другими словами, ориентация ребра должна совпадать с ориентацией соответствующего ему единичного вектора $e_{x}, x=1, \ldots, d$.

Определение 1. Будем говорить, что это ребро имеет метку $e_{x}$. 
Рассмотрим произвольную грань $\left(p_{1}, p_{2}, p_{4}, p_{3}\right) \in F(\mathscr{D})$. Пусть ребро $\left(p_{1}, p_{2}\right)$ имеет метку $e_{x}$, а ребро $\left(p_{1}, p_{3}\right)$ - метку $e_{y}, 1 \leqslant x, y \leqslant d, x \neq y$. Проверим, что

$$
\Psi\left(p_{4}, \lambda\right)+\alpha_{1}\left(p_{1}, p_{2}\right) \Psi\left(p_{2}, \lambda\right)+\alpha_{2}\left(p_{1}, p_{3}\right) \Psi\left(p_{3}, \lambda\right)+\alpha_{3}\left(p_{1}, p_{4}\right) \Psi\left(p_{1}, \lambda\right)=0,
$$

где коэффициенты $\alpha_{j}$ не зависят от $\lambda$ и определяются по следующим формулам:

$$
\begin{gathered}
\alpha_{1}\left(p_{1}, p_{2}\right)=-\lim _{\lambda \rightarrow A_{x}^{+}} \frac{\Psi\left(p_{4}, \lambda\right)}{\Psi\left(p_{2}, \lambda\right)}, \quad \alpha_{2}\left(p_{1}, p_{3}\right)=-\lim _{\lambda \rightarrow A_{y}^{+}} \frac{\Psi\left(p_{4}, \lambda\right)}{\Psi\left(p_{3}, \lambda\right)}, \\
\alpha_{3}\left(p_{1}, p_{4}\right)=-1-\alpha_{1}\left(p_{1}, p_{2}\right)-\alpha_{2}\left(p_{1}, p_{3}\right) .
\end{gathered}
$$

Действительно, левая часть в (1) удовлетворяет всем условиям для $\Psi(p, \lambda)$ за исключением того, что в $R_{1}$ она обращается в нуль. Тогда по теореме РиманаРоха сумма равна нулю при любом $\lambda \in \Gamma$.

Проделанные к этому моменту алгебро-геометрические построения аналогичны [2]. Займемся приведением формулы (1) к виду дискретного уравнения Коши-Римана.

Лемма 1. Пусть на Г существует голоморфная инволюиия $\sigma$ с двумя неподвижсными точками $R_{+}=R_{1}$ и $R_{-}$и выполняется равенство $\sigma A_{j}^{+}=A_{j}^{-} \partial л я$ $j=1, \ldots, d$. Кроме того, пусть существует мероморфный дифференциал $\Omega$ c двумя полюсами первого порядка в неподвижных точках $R_{+}, R_{-}$u $2 g$ нулями в $\gamma_{1}, \ldots, \gamma_{g}, \sigma \gamma_{1}, \ldots, \sigma \gamma_{g}$. Тогда

$$
\begin{gathered}
\Psi\left(p, R_{-}\right)=(-1)^{n_{1}(p)+\cdots+n_{d}(p)}, \\
\alpha_{1}\left(p_{1}, p_{2}\right)=-\alpha_{2}\left(p_{1}, p_{3}\right), \quad \alpha_{3}\left(p_{1}, p_{4}\right)=-1 .
\end{gathered}
$$

Хочется отметить, что многоточечная волновая функция вместе с уравнением (1) были построены еще в [8] при более общих предположениях. В этой работе они использовались для построения дискретного аналога решетки ДарбуЕгорова, размерность которой равна половине от количества неподвижных точек инволюции $\sigma$.

Пусть условия леммы выполнены. Определим $\nu$ на всех диагоналях граней из $F(\mathscr{D})$ по формулам

$$
\nu\left(p_{1}, p_{4}\right)=\frac{1}{i \alpha_{1}\left(p_{1}, p_{2}\right)}=\frac{i}{\alpha_{2}\left(p_{1}, p_{3}\right)}, \quad \nu\left(p_{2}, p_{3}\right)=\frac{1}{\nu\left(p_{1}, p_{4}\right)} .
$$

Тогда равенство (1) примет вид

$$
\frac{\Psi\left(p_{3}, \lambda\right)-\Psi\left(p_{2}, \lambda\right)}{\Psi\left(p_{4}, \lambda\right)-\Psi\left(p_{1}, \lambda\right)}=i \nu\left(p_{1}, p_{4}\right)=-\frac{1}{i \nu\left(p_{2}, p_{3}\right)} .
$$

Таким образом, волновая функция $\Psi(p, \lambda)$ является дискретной голоморфной на графе $\mathscr{D}$ с весовой функцией $\nu$ при любом фиксированном $\lambda \in \Gamma$.

В работе [7] неоднократно отмечается особый интерес случая положительной весовой функции. Для начала мы обсудим вещественность.

Лемма 2. Пусть выполнены условия леммы 1. Пусть на Г существует антиголоморфная инволюиия $\tau$ со следующими свойствами: $\tau \sigma=\sigma \tau, \tau R_{+}=R_{-}$, точки $A_{1}^{+}, A_{1}^{-}, \ldots, A_{d}^{+}, A_{d}^{-}$являются неподвижными относительно $\tau, \gamma$-дивизор как множество переходит в себя. Тогда весовая функция $\nu$ принимает вещественные значения и

$$
\Psi(p, \tau \lambda)=(-1)^{|\mathbf{n}(p)|} \overline{\Psi(p, \lambda)} .
$$


Проделанные к текущему моменту алгебро-геометрические построения аналогичны приведенным в [3]. Инволюция $\tau$ также используется и в [8] как условие вещественности решетки.

2. Достаточное условие несингулярности оператора Лапласа. Пусть выполнены условия лемм 1 и 2. Пусть также Г является М-кривой. Это означает, что антиголоморфная инволюция $\tau$ имеет $g+1$ неподвижных овалов $a_{1}, \ldots, a_{g}, c$. Потребуем еще, чтобы все выделенные точки попадали на один овал: $A_{j}^{ \pm} \in c, j=1, \ldots, d$. Отсюда следует, что $\sigma c=c$. При подходящей нумерации точек $A_{j}^{ \pm}$они располагаются на овале $c$ в порядке

$$
A_{1}^{+}, A_{2}^{+}, \ldots, A_{d}^{+}, A_{1}^{-}, A_{2}^{-}, \ldots, A_{d}^{-} .
$$

Будем считать, что именно такая нумерация была выбрана изначально.

Лемма 3. Пусть $\left(p_{1}, p_{2}, p_{4}, p_{3}\right) \in F(\mathscr{D})$ - произвольная грань. Не умаляя общности, будем считать, что ребра этой грани ориентированъ в сторону вершин с большими индексами (см. определение 1). Пусть ребро $\left(p_{1}, p_{2}\right)$ имеет метку $e_{x}$, а ребро $\left(p_{1}, p_{3}\right)$ - метку $e_{y}$. Тогда

$$
\lim _{A_{x}^{+}} \frac{\Psi\left(p_{2}, \lambda\right)}{\Psi\left(p_{4}, \lambda\right)} \frac{\Psi\left(p_{3}, \lambda\right)}{\Psi\left(p_{1}, \lambda\right)}>0, \quad \lim _{A_{y}^{+}} \frac{\Psi\left(p_{3}, \lambda\right)}{\Psi\left(p_{4}, \lambda\right)} \frac{\Psi\left(p_{2}, \lambda\right)}{\Psi\left(p_{1}, \lambda\right)}>0 .
$$

При доказательстве активно используется тот факт, что на овале $c$ волновая функция вещественна либо чисто мнима. Основным методом является подсчет вычетов дифференциалов вида $\Psi\left(\lambda, p_{i}\right) \Psi\left(\sigma \lambda, p_{j}\right) \Omega(\lambda)$ в выделенных точках.

Рассмотрим теперь две произвольные соседние грани $\left(p_{1}, p_{2}, p_{3}, p_{4}\right) \in F(\mathscr{D})$, $\left(p_{2}, p_{5}, p_{6}, p_{4}\right) \in F(\mathscr{D})$ квад-графа. Пусть ребро $\left(p_{1}, p_{2}\right)$ имеет метку $e_{x}$, ребро $\left(p_{2}, p_{4}\right)$ - метку $e_{y}$, ребро $\left(p_{2}, p_{5}\right)$ - метку $e_{z}$. Ориентация ребра $\left(p_{2}, p_{4}\right)$ может быть произвольной. Потребуем, чтобы ребра $\left(p_{1}, p_{2}\right)$ и $\left(p_{2}, p_{5}\right)$ имели общий конец или общее начало тогда и только тогда, когда точка $A_{y}^{+}$лежит между $A_{x}^{+}, A_{z}^{+}$на овале $c$. Назовем это условием С.

Теорема 1 (достаточное условие положительности весовой функции). Пусть выполняются описанные выше условия на спектральные данные. Все значения

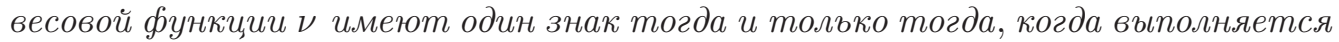
условие $\mathrm{C}$.

Будем доказывать положительность отношения значений $\nu$ на соседних гранях. При доказательстве необходимо разобрать три случая взаимной ориентации ребер $\left(p_{1}, p_{2}\right),\left(p_{2}, p_{5}\right)$. Для краткости разберем только тот, в котором они ориентированы в сторону вершин с большими индексами. Тогда

$$
\frac{\nu\left(p_{1}, p_{4}\right)}{\nu\left(p_{2}, p_{6}\right)}=\lim _{A_{x}^{+}} \frac{-\Psi\left(p_{2}, \lambda\right)}{\Psi\left(p_{4}, \lambda\right)} \lim _{A_{z}^{+}} \frac{\Psi\left(p_{6}, \lambda\right)}{-\Psi\left(p_{5}, \lambda\right)} .
$$

Рассмотрим мероморфную функцию $f(\lambda)=\Psi\left(p_{2}, \lambda\right) / \Psi\left(p_{4}, \lambda\right)$. По лемме 3 , примененной ко второй грани, левая часть последнего равенства больше нуля, если и только если $f\left(A_{x}^{+}\right) / f\left(A_{z}^{+}\right)>0$. Нетрудно видеть, что это эквивалентно тому, что точка $A_{y}^{+}$не лежит между $A_{x}^{+}, A_{z}^{+}$.

\section{ЛИТЕРАТУРА}

[1] Б. А. Дубровин, И. М. Кричевер, С. П. Новиков, Докл. АН СССР, 229:1 (1976), 15-18. [2] И. М. Кричевер, Докл. АН СССР, 285:1 (1985), 31-36. [3] A. Doliwa, 
P. Grinevich, M. Nieszporski, P. M. Santini, J. Math. Phys., 48:1 (2007), 013513. [4] А. П. Веселов, С. П. Новиков, Докл. АН СССР, 279:1 (1984), 20-24; 279:4 (1984), 784-788. [5] S. Grushevsky, I. Krichever, Duke Math. J., 152:2 (2010), 317-371. [6] J. Fay, Theta functions on Riemann surfaces, Lecture Notes in Math., vol. 352, Springer-Verlag, Berlin-Heidelberg-New York, 1973. [7] A. Bobenko, C. Mercat, Y. Suris, J. Reine Angew. Math., 583 (2005), 117-161. [8] А. А. Ахметшин, Ю. С. Вольвовский, И. М. Кричевер, в кн.: Труды МИАН, т. 225, Наука, М., 1999, 21-45.

Московский государственный университет им. М. В. Ломоносова

Поступила в редакцию e-mail: vasilevskiy.boris@gmail.com

11 ноября 2013 г.

\section{УДК $517.587+517.588$}

\section{Детерминантные меры, связанные с большими полиномами $\boldsymbol{q}$-Якоби*}

\section{(c) 2015. В. Е. Горин, Г. И. ОльшАнский}

Детерминантные меры образуют специальный класс вероятностных мер на пространствах локально конечных точечных конфигураций (см. обзор Бородина [1]). Ключевое свойство детерминантной меры состоит в том, что ее корреляционные функции всех порядков выражаются некоторым простым образом через одну функцию двух переменных, которая называется корреляционным ядром.

В работе Бородина и Ольшанского [2] изучалось семейство детерминантных мер, названных $z w$-мерами. Они связаны с задачей гармонического анализа на бесконечномерной унитарной группе $U(\infty)$, которая была поставлена в работе Ольшанского [12]. Аналоги zw-мер существуют также для других бесконечномерных классических групп и для бесконечномерных симметрических пространств (Ольшанский-Осиненко [14]). zw-меры играют фундаментальную роль в бесконечномерном гармоническом анализе, поскольку их скейлинговые пределы управляют спектральным разложением некоторых выделенных унитарных представлений.

С другой стороны, zw-меры являются красивыми комбинаторными объектами, у которых есть много общего с так называемыми z-мерами - некоторым частным случаем введенных Окуньковым мер Шура на разбиениях. Как и меры Шура, zw-меры допускают обобщение, в котором возникает дополнительный деформационный параметр, аналогичный дайсоновскому $\beta$-параметру в теории случайных матриц или непрерывному параметру в симметрических функциях Джека (Ольшанский [13]).

Наша цель - показать, что понятие zw-мер можно обобщить в другом направлении, а именно, у zw-мер существует (неочевидный) q-аналог. Наш первый результат состоит в том, что у « $N$-частичных $q$-Zw-мер» есть предел при $N \rightarrow \infty$ и предельная вероятностная мера является детерминантной мерой на некотором пространстве бесконечных точечных конфигураций. Мы нахо-

* Исследование Г. И. Ольшанского выполнено в ИППИ РАН за счет гранта Российского научного фонда (проект №14-50-00150). 